\title{
Activités
}

$12-2 \mid 2015$

Varia

\section{L'analyse des travails pour la conception en formation}

Contribution de l'ergonomie à l'orientation de la conception amont d'un environnement virtuel pour la formation

A works analysis approach for designing professional training. Contribution of ergonomics in orienting the upstream design of a virtual reality environment

\section{Vincent Boccara et Catherine Delgoulet}

\section{OpenEdition}

Journals

Édition électronique

URL : http://journals.openedition.org/activites/1098

DOI : 10.4000/activites. 1098

ISSN : $1765-2723$

Éditeur

ARPACT - Association Recherches et Pratiques sur les ACTivités

\section{Référence électronique}

Vincent Boccara et Catherine Delgoulet, «L'analyse des travails pour la conception en formation », Activités [En ligne], 12-2 | 2015, mis en ligne le 15 octobre 2015, consulté le 19 avril 2019. URL : http:// journals.openedition.org/activites/1098; DOI : 10.4000/activites.1098

\section{(c) (i) () $\Theta$}

Activités est mis à disposition selon les termes de la licence Creative Commons Attribution - Pas d'Utilisation Commerciale - Pas de Modification 4.0 International. 


\title{
L'analyse des travails pour la conception en formation.
}

\section{Contribution de l'ergonomie à l'orientation de la conception amont d'un environnement virtuel pour la formation $^{(*)}$}

\section{Vincent Boccara}

CNRS-LIMSI, Rue John Von Neumann, Bat. 508, F-91403 Orsay Cedex. Université Paris-Sud, Bat. 452, F-91403 Orsay Cedex - boccara@limsi.fr

\section{Catherine Delgoulet}

\author{
Université Paris Descartes, Laboratoire Adaptations Travail-Individus (LATI), 76 avenue Édouard Vaillant, \\ F-92550 Boulogne-Billancourt - catherine.delgoulet@ parisdescartes.fr \\ ${ }^{(*)}$ Cet article existe également en version anglaise dans ce numéro (pp. 137-158)
}

\section{ABSTRACTS}

A works analysis approach for designing professional training. Contribution of ergonomics in orienting the upstream design of a virtual reality environment. The aim of this article is to present and discuss a work analysis approach. This approach was developed within the context of a design project aiming to build a virtual environment for training (VET) workers involved in the assembly of aircraft parts. The frameworks of ergonomics and professional didactics served as a background for this study which took place in three stages: 1) an analysis of both demand and project, 2) work analysis (trainers, trainees and production unit workers, and 3) a co-analysis device to co-analyse with trainers the work knowledge and its transmission. During the study, the focal point of work analysis, the people concerned and the results produced by ergonomists all evolved and became more complex. Using reflection on our own ergonomic practice, we discuss the benefits of work analysis as a means of identifying the wide range of training situations and of taking part in design projects.

\section{KEYWORDS}

conception, training, virtual environment, analysis of works, aeronautics

Cet article propose une réflexion sur une démarche d'analyse des travails en vue de participer à la conception d'un outil de formation. Plus précisément, nous nous focaliserons sur comment cette démarche contribue à l'orientation des choix de conception d'un démonstrateur d'environnement virtuel de formation. Il a ainsi pour objectif de contribuer à la production de modèles pour l'intervention des ergonomes en conception et plus particulièrement dans le domaine de la formation professionnelle. Il prend sa source dans un projet de recherche pluridisciplinaire (réunissant un industriel et un site de sa filiale, des informaticiens, automaticiens, designers et ergonomes) de conception amont d'un 
environnement virtuel de formation (EVF) au métier d'assembleur-monteur aéronautique ${ }^{1}$. En tant qu'ergonomes, notre cahier des charges initial comporte deux volets : 1) l'analyse des situations professionnelles existantes en vue d'une scénarisation de situations d'apprentissage dans l'EVF et, 2) l'évaluation de la «pertinence interne et externe » (Delgoulet, 2001) de l'EVF pour apprendre un métier particulier. Seul le premier volet est exposé ici.

Ce projet renvoie ainsi aux premières phases du processus de l'élaboration d'un outil, celles de la conception d'un démonstrateur selon deux versions : entre deux dimensions selon un format «écran-clavier», ou en trois dimensions selon une modalité «immersive». Ce démonstrateur est destiné à s'insérer dans des dispositifs de formation professionnelle existants ; outil ayant a priori vocation à devenir un instrument ${ }^{2}$ de formation pour les formateurs aussi bien qu'un instrument d'apprentissage pour les formés, futurs professionnels. Cette finalité appelle, de notre point de vue, à mettre en place une analyse du travail au sens de Daniellou (1996, p. 185), c'est-à-dire « la démarche globale, où l'analyse de l'activité est insérée dans une analyse des facteurs économiques, techniques et sociaux auxquels l'opérateur ou l'opératrice est confronté, et une analyse des traces du fonctionnement de l'entreprise sur la population concernée et l'efficacité économique ». Dans cette perspective, nous avons construit une démarche d'analyse "des travails » des formateurs, des formés et d'opérateurs de production à la croisée des approches défendues en ergonomie et didactique professionnelle (Boccara \& Delgoulet, 2013 ; Chatigny \& Vézina, 2008 ; Olry \& Vidal-Gomel, 2011 ; Ouellet, Vézina, \& Chatigny, 2013 ; Pastré, 2011).

La démarche proposée questionne ainsi la place de l'analyse du travail, son périmètre et les acteurs impliqués pour que l'ergonome participe à la conception d'un outil pour la formation (définition des objectifs, dispositifs et situations de formation, propriétés de l'environnement virtuel et des objets manipulés, fonctionnalités de l'outil, etc.). Sans entrer dans le détail de l'ensemble des analyses réalisées, nous discutons ici de l'analyse «des travails» comme moyen d'identifier les multiples horizons des situations de formations à concevoir : «à quoi former ? », « comment former ? », « quel(s) dispositif(s) ? », « pour quels usages ? (Olry \& Vidal-Gomel, 2011) en considérant que les conditions de travail en formation sont des conditions d'apprentissage pour les formés (Chatigny \& Vézina, 2008 ; Ouellet, Vézina, \& Chatigny, 2013). En conclusion, nous revenons sur les apports potentiels de cette démarche aux modèles de l'intervention en ergonomie dans le champ de la conception de formation.

\section{1.- Cadrage théorique de la démarche}

La conception d'EVF fait l'objet de nombreux travaux dont les réflexions portent notamment sur les technologies supports (RV non immersive, RV immersive ou Réalité Augmentée), les informations manipulées dans ou par ces outils et leurs modalités de présentation (e.g. Brough, Schwartz, Gupta, Anand, Kavetsky, \& Pettersen, 2007 ; Costello, 1997). Plus rarement, les analyses sont centrées sur les utilisateurs (essentiellement les formés) et leurs besoins (Anastassova, Burkhardt, Mégard, \& Ehanno, 2007) et considèrent les tâches telles que prescrites en situation de travail (e.g. Hu, Zhang, \& Salvendy, 2012). L'activité et ses conditions d'élaboration en situation sont quasiment absentes, induisant une approche de l'apprentissage basée sur des présupposés ou des prescriptions qui ne permettent pas de considérer la variabilité et la diversité des situations réelles d'accomplissement des tâches et leur transposition en situation apprentissage.

1 Projet de recherche Natural Interactions, Knowledge, Immersive system for Training in Aeronautic (NIKITA) financé par l'Agence National pour la Recherché (ANR) et coordonné par Domitile Lourdeaux du laboratoire Heudiasyc de l'Université Technologique de Compiègne (http://www.emissive.fr/nikita/).

2 Au sens de Rabardel (1995, p. 74), l'instrument est « une entité mixte [...] une totalité comprenant à la fois un artefact (ou une fraction d'artefact) et un ou des schèmes d'utilisation. » 
En ergonomie de l'activité et didactique professionnelle, la conception de formations prend classiquement pour référence les situations réelles de travail (Haradji, Kostulski, Morais, \& Ughetto, 2014). Cependant, lors de la conception d'un nouvel outil de formation comme c'est le cas ici, nous faisons l'hypothèse que ces situations ne peuvent être la seule référence si l'on souhaite prendre en compte dès les phases amont de la conception l'ensemble des acteurs et des situations susceptibles d'être concernés par le changement d'outil envisagé. Il s'agit alors plutôt d'identifier les situations de référence (Samurçay \& Rogalski, 1998) pertinentes pour l'anticipation des situations futures probables afin d'orienter et agir durant le processus de conception du nouvel outil de formation (Daniellou, 2004). La conception visant la formation, ces situations de références sont dans deux domaines : 1) le travail en production et 2) le travail en formation.

\section{1.- L'analyse de l'activité pour la conception de formations}

Depuis les premiers travaux issus de l'ergonomie de l'activité (Leplat, 1955 ; Montmollin de, 1974 ; Ombredane \& Faverge, 1955 ; Pacaud, 1975), les chercheurs ont contribué à alimenter le champ de la formation professionnelle (pour une revue plus complète, voir : Teiger \& Lacomblez, 2013). De manière prudente et sporadique pendant longtemps, les travaux se sont multipliés au tournant des années 90 (Rabardel, Teiger, Laville, Rey, \& Desnoyers, 1991; Teiger \& Montreuil, 1996). Leurs contributions à la conception de formations sont aujourd'hui nombreuses et peuvent être organisées autour de quatre grands thèmes :

1. L'analyse de l'activité en situation de travail pour identifier les objectifs de formation et les compétences critiques auxquelles il faudrait former pour outiller les travailleurs (Berglund \& Karltun, 2012 ; Chatigny \& Vézina, 1995 ; Leppänen, 2001 ; Montmollin de, 1974 ; Ouellet \& Vézina, 2008), concevoir des outils pédagogiques adaptés aux besoins des futurs formés et des formateurs (Anastassova, Burkhardt, Mégard, \& Ehanno, 2005 ; Aubert-Blanc, 2009 ; Boccara \& Delgoulet, 2013 ; Olry \& Vidal-Gomel, 2011), identifier les compétences acquises, transférables dans une perspective de reconversion professionnelle (Rabardel \& Six, 1995), identifier les difficultés d'appropriation au travail de savoirs et savoir-faire vus en formation et faire une évaluation rétrospective des formations (Delgoulet, 2001 ; Gaudart, 2000) ;

2. L'analyse de l'activité d'apprentissage pour identifier les facilités et difficultés rencontrées par les apprenants dans leur travail d'appropriation des savoirs (Delgoulet \& Marquié, 2002 ; Santos \& Lacomblez, 2007 ; Cau-Bareille, Gaudart, \& Delgoulet, 2012) ;

3. L'analyse de l'activité de transmission/partage des savoirs et savoir-faire en situation de travail (tutorat, compagnonnage, etc. ; Cloutier, Fournier, Ledoux, Gagnon, Beauvais, \& Vincet-Genod, 2012; Delgoulet, Largier, \& Tirilly, 2013; Gaudart, Delgoulet, \& Chassaing, 2008 ; Thébault, Gaudart, Cloutier, \& Volkoff, 2012 ; Thébault, Delgoulet, Fournier, Gaudart, \& Jolivet, 2014) ou en situation de formation initiale ou continue (Anastassova \& Burkhardt, 2009 ; Messing, Escalona, \& Seifert, 1996 ; Rogalski, 2003 ; Veyrac \& Asloum, 2009; Viau-Guay, 2014; Vidal-Gomel, Boccara, Rogalski, \& Delhomme, 2012) pour caractériser les interactions entre formateurs/enseignants et formés, proposer des outils pédagogiques adaptés aux situations et aux besoins, ou des aménagements organisationnels facilitant l'activité conjointe d'apprentissage en situation de travail ou de formation;

4. La formation à et par l'analyse de l'activité de travail d'acteurs de la prévention de la santé et des risques professionnels ou des représentants des salariés pour transformer leurs représentations du travail et des actions à y engager (Gaudart et al., 2012 ; Hubault, Noulin, Dupont, \& Mélier, 1994 ; Teiger \& Lacomblez, 2013 ; Teiger \& Laville, 1991 ; Wendelen, 1996); ces travaux ayant également donné lieu à une réflexion sur l'évaluation de tels programmes et leurs retombées (Berthelette, 1995 ; Desnoyers, 
Berthelette, Gourde, \& Authier, 1997 ; Lang, 2013 ; Montreuil, Brisson, Arial, \& Trudel, 1997).

Notre contribution s'inscrit à la croisée des trois premiers thèmes en proposant une démarche d'analyse de l'activité de travail et d'intervention de l'ergonome dans le champ de la conception de formation professionnelle. Toutefois, l'originalité de notre positionnement réside notamment dans la manière dont la situation de formation est considérée comme une situation de travail à part entière. Ce postulat se base sur trois arguments complémentaires : 1) les formateurs sont des travailleurs pris dans des enjeux socio-économiques de viabilité, qualité, performance des dispositifs de formation qu'ils peuvent concevoir, coordonner et/ou animer ; leur activité est soumise à des contraintes et répond à des exigences, tout autant que celle des opérateurs en production; 2) les formés en formation professionnelle sont des salariés d'une entreprise (de travail temporaire ou du secteur de l'aéronautique); leur activité peut ainsi être appréhendée comme un «travail d'apprentissage » (Montmollin, 1980) qui nécessite d'être relié à son contexte de réalisation; 3) ces travails en formation (des formateurs et formés) questionnent et doivent orienter la conception de formation comme peut le faire de manière plus «évidente » le travail des opérateurs en production.

Il s'agit alors de notre point de vue de mettre en place une démarche d'analyse du travail qui permette de prendre en compte les situations de référence pertinentes en production et en formation pour concevoir un nouveau dispositif ou outil susceptible de transformer les conditions de réalisation d'une formation. Ainsi, le périmètre de l'analyse ergonomique du travail comprend aussi bien les situations de production visée à l'issue de la formation que les situations de formation elles-mêmes (Boccara \& Delgoulet, 2013 ; Beaujouan, Aubert, \& Coutarel, 2013).

\section{2.- L'analyse conjointe de l'activité de travail des formateurs et des formés}

Le travail des formateurs a longtemps été délaissé par les ergonomes et psycho-ergonomes, qui lorsqu'ils s'intéressaient aux activités de transmission ou d'accompagnement des actifs dans la construction des savoirs, s'occupaient davantage d'ergonomie scolaire du point de vue des apprenants. Des travaux se sont ainsi intéressés aux rythmes scolaires (Delvolvé \& Davila, 1994), à l'adaptation ou la conception du poste et des outils de travail des enfants (Jacobs \& Backer, 2002), à l'activité des enseignants en classe (Amigues, 2003 ; Faïta, 2003 ; Ria, Sève, Durand, \& Bertone, 2004 ; Rogalski, 2003, 2004) et à leurs conditions de travail (Jobert, 1987 ; 1993 ; 2000 ; Méhaut, 1995) et leurs effets sur la santé (Cau-Bareille, 2011 ; Chatigny, Levesque, \& Riel, 2012 ; Delgoulet, 2012 ; Riel \& Messing, 2011 ; Santos $\&$ Feirrera, 2012).

Nous nous appuierons ici sur le modèle de l'activité de l'enseignant proposé par Rogalski (2003) pour appréhender l'activité de travail des formateurs d'un triple point de vue : professionnel, didactique et la gestion d'un environnement dynamique ouvert (Boccara, 2011 ; Vidal-Gomel et al., 2012). Toutefois, notre approche vise à dépasser une analyse de l'activité des formateurs faite indépendamment de celle des formés ou bien complétée d'une analyse de l'activité de(s) formés. Il s'agit plutôt d'essayer d'appréhender conjointement l'activité de travail des formateurs et des formés dans la perspective de la double approche (Rogalski, 2012), basée sur le modèle de double régulation de l'activité de travail (Leplat \& Cuny, 1984). Schématiquement, cette proposition tente de dépasser les approches analysant les situations de formation du point de vue du formateur/enseignant et/ou du formé séparément ou dans une perspective interactionniste en se plaçant dans la perspective de l'activité déployée par les acteurs de la situation : formateur(s) et formé(s).

Dès lors, cette approche considère que les déterminants de la situation sont des déterminants de l'activité du formateur et des formés, ce qui rejoint les propositions des travaux québécois considérant les conditions de travail en formation comme des conditions d'apprentissage 
(Chatigny \& Vézina, 2008 ; Ouellet \& Vézina, 2008 ; 2009 ; Ouellet et al., 2003). De plus, elle considère également que l'activité du (des) formé(s) est un déterminant de l'activité du (des) formateurs, et réciproquement. L'activité formateur-formé peut ainsi être considérée comme une co-construction située, dynamique, évolutive, protéiforme, multidimensionnelle et multifonctionnelle. Elle présente ainsi des caractéristiques communes (sans s'y limiter) avec les interactions formatives constitutives de la transmission professionnelle ${ }^{3}$ telles que définies par Thébault (2013) : c'est-à-dire « les interactions qui cherchent à agir sur autrui, qui ont pour objectif de le former, de participer à son développement» (p. 115) en situation de production ou de service.

\section{3.- Des situations d'action caractéristique à leur transposition didactique}

En ergonomie et en didactique professionnelle, la conception de formation s'appuie sur l'identification et l'analyse de situations d'action caractéristiques (Daniellou, 2004) pour préconiser des objectifs de formation (Olry \& Vidal-Gomel, 2011; Chatigny \& Vézina, 2008 ; Ouellet, Vézina \& Chatigny, 2013 ; Beaujouan, Aubert, \& Coutarel, 2013), à partir de la formalisation de savoirs professionnels de référence (Samurçay \& Rabardel, 2004) considérés comme les contenus auxquels il faudrait former du point de vue du travail. Certains travaux ont complété cette approche en analysant également en amont de la conception de formation les déterminants des situations de travail en considérant que l'amélioration des conditions de travail des formateurs améliore les conditions d'apprentissage des formés (Ouellet, Vézina \& Chatigny, 2013 ; Ouellet \& Vézina, 2008, 2009 ; Ouellet et al., 2003).

Cependant, plus rares sont les recherches qui analysent les transpositions didactiques (Olry \& Vidal-Gomel, 2011 ; Samurçay \& Rogalski, 1998) qui sont à l'œuvre dans les situations existantes de formation par rapport à celles de «production». Il semble pourtant important d'identifier les écarts et les proximités de ces deux types de situations du point de vue des activités qui s'y déploient, de celles qui ne peuvent pas s'y déployer et de celles qu'ils seraient souhaitables de s'y déployer pour favoriser les apprentissages des formés et le développement de leur activité. En fait, l'analyse de ces écarts peut orienter la conception de formation et plus particulièrement celle d'un nouvel outil de formation, dont l'introduction est susceptible de modifier les conditions de réalisation de l'activité formateur-formé. Ainsi, si l'on considère que ce type de projet renvoie à la conception d'un nouvel artefact à vocation instrumentale (Rabardel, 1995) pour les formateurs et les formés, l'analyse de ces écarts est une des pistes potentielles pour l'ergonome en vue d'interroger le souhaitable et le possible au cours de la conception (Daniellou, 2004). Le projet d'un nouvel artefact étant potentiellement source d'ouverture du champ des possibles pour l'activité formateur(s)formé(s), aussi bien que de contraintes susceptibles de l'enfermer, voire de l'empêcher (Clot, 2000), il s'agit pour l'ergonome de veiller à favoriser le premier versant sans alimenter la voie de l'enfermement.

Dans cette perspective, l'objectif de la démarche d'analyse des travails vise à produire un référentiel de situations pour la formation (Mayen, Métral, \&Tourmen, 2010). Ce référentiel serait un objet intermédiaire du projet de conception (Jeantet, 1998 ; Vinck, 2009), dont l'élaboration peut être poursuivie dans l'usage par les formateurs après le temps de la conception. L'idée poursuivie ici est que ce référentiel pourrait à terme devenir un des instruments des formateurs pour la construction de situations potentielles de développement pour les formés. En cela, la démarche proposée vise à soutenir elle-même le développement chez les acteurs qu'elle implique.

3 «Ensemble d'échanges à propos des pratiques professionnelles, au cours de la réalisation du travail, entre des individus aux parcours, expériences et anciennetés divers, échanges marqués par les asymétries que cette diversité implique entre ces individus » (p. 112) 


\section{2.- Démarche et méthodologie d'investigation}

\section{1.- La démarche d'analyse des travails}

La démarche mise en œuvre comprend trois phases. Elles visent à construire, préciser les objectifs du projet de recherche en collaboration avec l'ensemble des partenaires et à considérer dans leurs particularités les trois situations de référence déjà identifiées de manière générique. Ces trois phases renvoient à (cf. Figure 1) :

- une analyse et reformulation de la demande : classique en ergonomie, mais beaucoup moins dans ce genre de projet, elle permet d'impliquer les acteurs stratégiques sur le site partenaire (en production, prévention, formation ou dans le cadre du $\mathrm{CHSCT}^{4}$ du site) ; de discuter de l'intérêt, des conditions et des possibilités d'accès aux situations réelles en production et en formation; dans le cas présent, elle intégrait une analyse du projet, son histoire, ses structures et sa conduite ;

- des analyses du travail des formateurs, des formés et des opérateurs de production, menées au niveau organisationnel et situationnel ; il s'agit de travailler les questions de la transposition entre travail en production et travail en formation d'une part; et, d'autre part, de nous intéresser à la période sensible de la prise de poste en atelier de production, entre travail en formation et travail en production ;

— une co-analyse des savoirs de métier et de leurs transmissions ${ }^{5}$ dans le cadre d'un dispositif méthodologique impliquant les formateurs. Cette troisième phase participe de l'analyse plus générale des travails en s'arrêtant sur une dimension centrale pour la conception d'un EVF, celle des compétences professionnelles du métier et leur développement. Deux objectifs guident cette dernière phase : a) mieux comprendre les compétences critiques sous-jacentes à l'activité des assembleurs-monteurs ; b) offrir une opportunité aux formateurs de développer leur propre activité en tant que formateurs en provoquant des activités réflexives sur ces compétences critiques et leur développement en formation (Weill-Fassina \& Pastré, 2004).

L'ensemble permet de construire et d'enrichir progressivement des repères pour la conception sur lesquels l'ergonome peut communiquer et s'appuyer pendant la vie du projet pour interagir avec les partenaires chargés de l'implémentation effective des scénarios, des propriétés des éléments manipulés dans l'environnement virtuel et des phénomènes physiques associés. Ici, ces repères pour la conception ont pris la forme d'un référentiel de situations de formation dans et avec l'EVF.

L'analyse des travails est utilisée d'une part comme un moyen de compréhension de l'activité des différents acteurs et d'identification des savoirs de métier et de leur développement en formation et, d'autre part, comme une ressource pour intervenir à la fois dans les situations de la formation professionnelle existante et pendant la conception d'un nouvel outil de formation, ici un EVF.

4 Comité Hygiène Sécurité et Conditions de Travail

5 Le terme de «transmission » rend compte ici de l'activité conjointe des formateurs et des formés en situation, orientée par le développement des compétences du formé. 


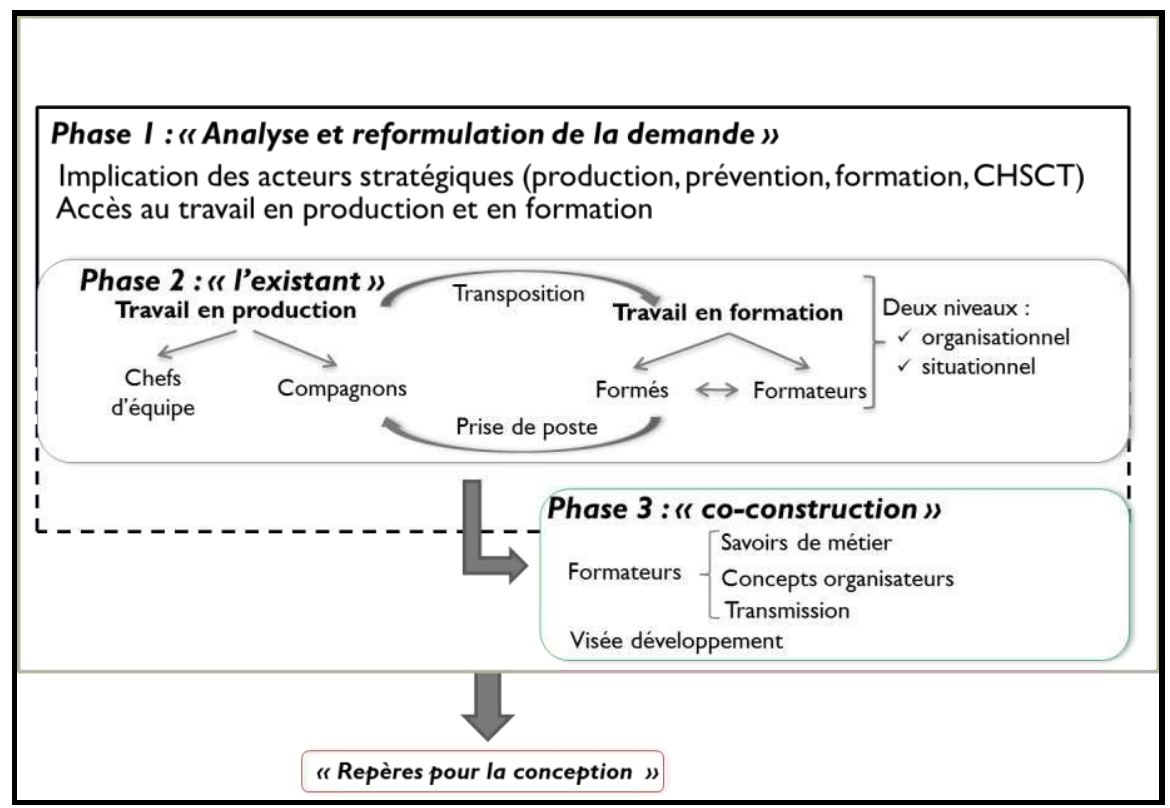

Figure 1 : Schématisation des trois phases de la démarche d'analyse des travails pour l'élaboration de repères pour la conception et des sous-objectifs poursuivis dans chacune d'elles

Figure 1: Schematization of the three phases of the work analysis approach

\section{2.- La situation de référence}

Un site de production et de formation (initiale et continue) de la filiale du partenaire industriel est identifié dans le projet comme site de référence. Il est spécialisé dans l'assemblage de tronçons avant d'avions (fuselages avant et cockpits). Environ 1500 salariés travaillent au quotidien sur le site de production. La population des «compagnons » (les monteurs-assembleurs), pour l'essentiel des hommes, est composée de deux groupes d'âges relativement contrastés formant un profil démographique bimodal (les moins de 30 ans et les plus de 45 ans). L'ancienneté des compagnons est largement liée à l'âge et évolue en parallèle. L'entrée dans le métier se fait majoritairement en début de vie professionnelle en tant que salarié permanent de l'entreprise à la suite d'une formation initiale (de niveau Bac professionnel) dispensée au centre de formation du site. Elle peut aussi prendre la forme d'un contrat intérim comprenant une formation professionnelle de dix semaines, dont huit semaines en centre de formation et deux semaines de tutorat sur un poste de production. Après validation de leur formation par l'obtention d'un Certificat de Qualification Professionnelle de la Métallurgie (CQPM), ces intérimaires peuvent devenir des salariés permanents à la suite d'une ou plusieurs périodes de travail intérimaire.

Les formés ${ }^{6}$, accueillis dans le cursus de formation continue, sont à $96 \%$ des hommes et constituent une population hétérogène du point de vue de l'âge, l'expérience professionnelle, la formation initiale et le niveau de diplôme. Deux tiers des formés sont des jeunes de moins de 25 ans ; les plus de 30 ans représentant $19 \%$ des formés. Parmi les formés, $15 \%$ ont un $\mathrm{BAC}+2$ d'une filière technique (BTS), $47 \%$ ont une Baccalauréat (professionnel ou technique) et $33 \%$ ont un CAP ou un BEP, voire dans certains cas ces deux diplômes. Les formés ont généralement une formation initiale en dehors du secteur de l'aéronautique, principalement la mécanique.

Dans cette entreprise, le rôle de «formateur » n'est pas structuré en métier, c'est plutôt une

6 Cette analyse a été conduite sur une population de 163 formés ayant suivi la formation entre 2010-2012. 
fonction qu'on occupe à temps plein ou à temps partiel. Ce rôle n'est pas non plus formellement intégré dans les parcours professionnels des compagnons du site de production comme ceci peut l'être dans d'autres entreprises (un moyen de reclassement et/ou d'évolution en fin de carrière; un moyen de débuter sa carrière). Au contraire, l'entité de formation rencontre même des difficultés pour « extraire » des compagnons des situations de production pour qu'ils deviennent formateurs intermittents ou permanents. Plus spécifiquement, la formation est coordonnée et animée par deux formateurs âgés de plus de 50 ans, proches de la retraite. Ils ont tous deux plus de 30 ans d'expérience du métier en production (en tant que compagnon sur les lignes de production et préparateur). L'un est formateur depuis deux ans et l'autre un an. Ils n'ont pas suivi de formation au métier de formateur qu'ils ont appris sur le tas par compagnonnage avec les formateurs précédents.

Pour faire face à un besoin accru de main d'œuvre, le site de production sollicite fortement le centre de formation via ses deux filières d'enseignement (initial ou professionnel).

\section{3.- Le recueil des données}

Ce travail s'appuyant sur une approche majoritairement qualitative à partir de matériaux empiriques issus de situations réelles de travail ou de formation, nous avons opté pour une triangulation de méthodes (Guilbert \& Lancry, 2007; Jick, 1979; Leplat, 2002). Cette triangulation consiste à combiner plusieurs méthodes de recueil de données pour : 1) réduire les biais inhérents à chaque méthode et augmenter ainsi la fiabilité et la validité du recueil empirique; 2) fournir une richesse qualitative et une meilleure compréhension du phénomène étudié ; 3) faciliter l'appréhension de processus qui ne sont pas directement observables, comme la conceptualisation dans l'action (Pastré, 1999). Cette démarche combine ainsi des méthodes classiques en ergonomie : des observations de terrain (Guérin, Laville, Daniellou, Duraffourg, \& Kerguelen, 1991 ; Rabardel, Carlin, Chesnais, Lang, \& Pascal, 1998), des entretiens exploratoires (Blanchet \& Gotman, 1992) ou d'autoconfrontations (Clot, Faïta, Fernandez, \& Scheller, 2000 ; Falzon \& Mollo, 2004) et, une collecte documentaire (Guérin, et al., 1991). La démarche d'analyse des travails mise en œuvre repose ainsi sur un total de quatorze jours de terrain - huit en formation et six en production -, de vingt entretiens semi-directifs et de deux séances d'auto-confrontation de quatre heures chacune - une avec chaque formateur. Le corpus empirique ainsi constitué provient de sources de recueil variées. C'est pourquoi un journal de terrain (Arborio \& Fournier, 1999) a été rédigé tout au long du projet afin de tenir la cohérence de l'objectif général d'analyse des travails pour l'orientation du projet, puis la conception amont d'un EVF. Ce journal permet de reconstituer la chronologie des événements constitutifs du projet de conception (Boccara \& Delgoulet, 2013), mais aussi de s'assurer de l'articulation temporelle des recueils et des contenus produits.

En formation, les journées d'observations ont été réalisées de manière à avoir une vue d'ensemble de la formation professionnelle dédiée aux travailleurs intérimaires lors de leur passage sur la ligne-atelier. Des entretiens exploratoires ont été réalisés avec les deux formateurs en fonction, pour cerner et comprendre leur travail. D'autres ont été menés avec 20 formés issus de trois groupes distincts, à un moment particulier de leur parcours d'apprentissage sur la ligne-atelier : un en début, un en milieu et un en fin de formation. Ces entretiens avaient pour objectifs de retracer leur parcours professionnel, repérer leur(s) évolution(s) dans la formation et les facilités/difficultés d'apprentissage perçues. Un ensemble de documents a également été collecté et analysé : le programme de formation, les supports de cours magistraux et les fiches techniques des exercices sur la ligne d'atelier.

En production, deux postes de travail ont été sélectionnés en concertation avec la direction du centre de formation, l'ergonome du site et le responsable de la production : le cockpit d'un premier type d'avion, désigné sous le terme de « la pointe avant», et le fuselage avant 
d'un second, appelé «barque $»^{7}$. Ces acteurs considèrent que ces deux postes représentent de manière satisfaisante les principales caractéristiques des tâches de montage-assemblage qu'un compagnon est amené à réaliser. Ils permettent d'une part d'appréhender la diversité des situations (opérations, fixations, pièces, types d'avion) et des cadences de production (selon le type d'avion assemblé), la présence d'aléas (approvisionnement, effectifs au poste, encombrement des espaces de travail, accessibilité des éléments à fixer, etc.), la dimension collective du travail (partage d'espaces de travail, d'outils et réalisation de tâches à deux). Ils répondent d'autre part aux exigences et attentes de l'entreprise en termes de protection $\mathrm{du}$ secret industriel, de continuité de la production ${ }^{8}$, d'opérations pérennes dans les plans prévisionnels de production (pas de sous-traitance envisagée dans l'avenir) et de contraintes professionnelles, notamment physiques, inhérentes au métier. Nous avons également recueilli un ensemble de documents relatifs aux données de description de la population et des accidents du travail, produites ou collectées par les services des ressources humaines et de la prévention des risques professionnels. Pour des raisons de secret industriel, nous avons seulement pu consulter sur place les documents de prescription et de traçabilité du travail des compagnons : fiches de postes, plan de production, fiches techniques et fiches suiveuses des tâches de montage-assemblage.

Le dispositif de co-construction déployé en phase 3 est mené exclusivement avec les formateurs. Elle consiste à : 1) choisir une tâche existante que les formateurs réaliseront ; 2) filmer les formateurs en train de réaliser la tâche ; 3) mener un entretien d'autoconfrontation avec chacun d'eux.

La tâche (exercice 1), choisie en accord avec les deux formateurs, présente l'avantage de couvrir un spectre large de sous-tâches, procédés et outils du métier; elle peut être réalisée sur une journée sans gêner l'accueil d'un groupe de formés sur la ligne-atelier. Deux caméras ont été utilisées pour filmer chaque formateur sur une journée $(16 \mathrm{~h}$ de vidéo au total) : l'une, subjective, permet d'apprécier le champ de vision du formateur durant la réalisation de la tâche et d'être au plus près des dimensions visuelles fines qui constituent le métier; l'autre, externe, filme le même formateur et son environnement de travail. Un montage en parallèle de ces deux enregistrements permet d'appréhender de manière globale la scène qui se déroule et, facilite les échanges ultérieurs lors des auto-confrontations (cf. Figure 2). Durant l'entretien, le formateur a comme consigne de regarder librement la séquence vidéo (possibilité de ralentir/accélérer ou d'arrêter) en commentant les éléments qui lui semblent importants. Les arrêts sont des occasions pour relancer le formateur sur les visées de transmission aux formés et leurs modalités pratiques. Ces entretiens d'autoconfrontation ont été filmés, puis retranscrits. Leur objectif était notamment d'appréhender la dimension conceptuelle de l'activité des assembleur-monteurs (Pastré, 1999, 2005, 2011), dans la réalisation de quatre tâches élémentaires du métier (perçage, contre-perçage, alésage et rivetage), centrales en formation.

7 En raison d'une absence temporaire de production au poste "pointe avant" lors de notre période de recueil empirique, la plupart des observations ont été réalisées sur le second poste.

8 La présence au poste d'un ou deux chercheurs ne devait pas gêner le travail des compagnons. Les espaces, parfois restraints au poste de travail, ne permettaient pas toujours d'accueillir des observateurs. 


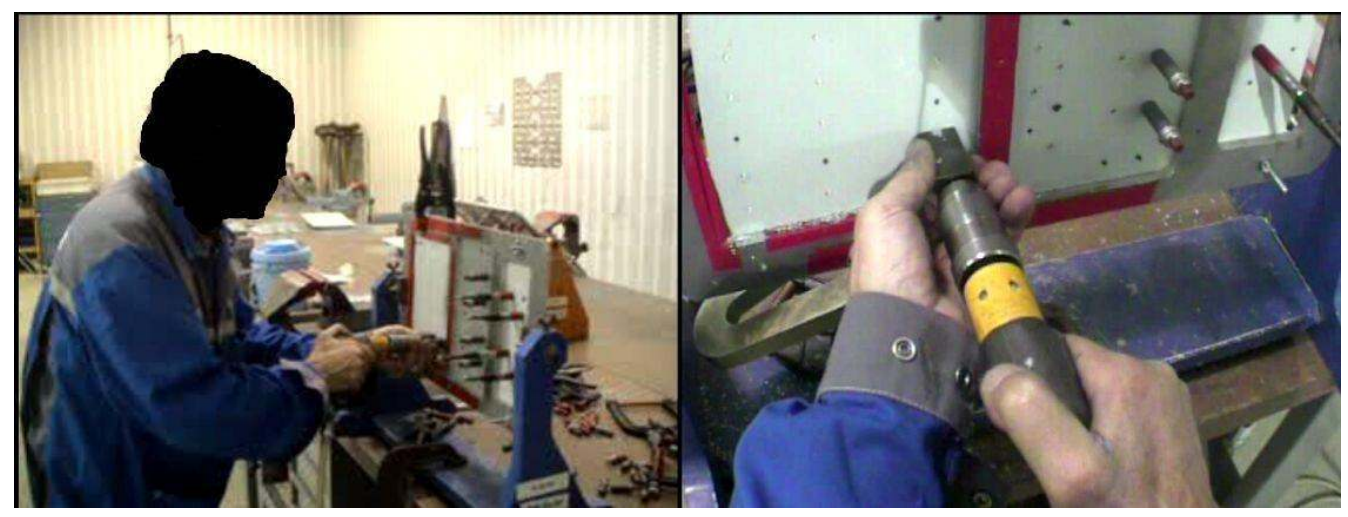

Figure 2 : Exemple de rendu vidéo après montage des films issus des deux caméras (Externe : à gauche, Subjective : à droite)

Figure 2: An example of video playback after editing film from the two cameras (external on the left and subjective on the right)

\section{3.- Au-delà de l'analyse de l'existant en production}

\section{1.- L'analyse de la demande initiale et sa reformulation : vers une approche plus globale et partagée}

L'ambition du projet est de proposer un environnement virtuel assurant un équilibre entre trois dimensions (Amokrane, K., Andriot, C., Boccara, V., Carpentier, K., Convard, T., Delgoulet, C. et al., 2013) : a) la cohérence de l'environnement perçu: interactions naturelles, environnements virtuels réalistes aux sens graphique, physique et sémantique ; b) l'émergence: liberté d'action, confrontation à une grande diversité de situations dans un environnement sociotechnique complexe; c) le contrôle: adaptation dynamique, personnalisation des situations d'apprentissage et des assistances à l'interaction en fonction des objectifs de formation, des intentions et du profil de l'apprenant. Sous cet objectif général, tourné essentiellement vers la levée de «verrous »" scientifico-technologiques, de nombreux enjeux se font pourtant jour.

Il n'est pas question ici de les lister de manière exhaustive, mais de donner une idée de leur diversité. Par exemple, le partenaire industriel, déjà doté d'un service dédié à la Réalité Virtuelle (RV), a ainsi fait le pari depuis quelques années de l'apport de ces technologies pour le maintien et l'accroissement de sa compétitivité. Son implication dans le projet vise à mesurer, voire valider, la plus-value de la RV pour former des assembleurs-monteurs. Le centre de formation considère ce projet comme une opportunité de positionnement novateur sur le marché de la formation et de réduction des coûts de formation. Pour le service de prévention, c'est l'occasion de (re)questionner la conception actuelle des postes et les causes d'accidents (notamment chez les novices). L'intérêt, formulé par le service des méthodes, réside dans une familiarisation plus précoce des formés aux contraintes physiques, organisationnelles des situations professionnelles ainsi qu'aux exigences de performance et de qualité. Dans un autre registre, l'équipe de recherche chargée de la scénarisation souhaite notamment avancer sur des questions d'intégration, dans leur modèle d'animation des Personnages Virtuels Autonomes (PVA), de dimensions peu traitées jusqu'à présent dans la littérature : la restitution de la dimension collective, liée à la coordination de l'action en situation entre PVA et formé ; ou celle des émotions face à des situations inattendues, etc.

En référence aux travaux de Daniellou (2004; 1992), la fonction de Maîtrise d'OuvrAge

$9 \quad$ Terme employé systématiquement par l'ANR pour qualifier les défits et enjeux scientifiques. 
(MOA) comporte deux niveaux d'acteurs dans un tel projet, l'ANR et le partenaire industriel. En tant que financeur, l'ANR peut être considérée comme une première partie de la maîtrise d'ouvrage porteuse d'une «volonté relative au futur » concernant l'orientation des objets prioritaires de la recherche scientifique nationale dans le développement d'une économie de l'innovation et de la connaissance (MESR, 2009). Le partenaire industriel et le centre de formation portent également pour partie une «volonté relative au futur» concernant les usages souhaités d'un tel EVF pour la formation professionnelle des opérateurs de production. La Maîtrise d'E Euvre (MOe) - porteuse de la faisabilité du projet est constituée des trois équipes de recherche (dont font partie les ergonomes) et de l'entreprise de graphisme 3D. Chacune est responsable et garante de la réalisation d'une partie technico-scientifique du futur système ; l'une d'entre elles assure le pilotage projet, notamment pour l'ANR.

Assez classiquement, force est de constater un manque d'identification claire et une «faible» présence de la MOA dans le projet au profit de la MOe, «le projet est alors conduit par la technique »(Daniellou, $2004 ;$ p. 361). La place initialement prévue ${ }^{10}$ pour l'équipe d'ergonome s'apparente à une partie de la MOe en charge de l'intégration des facteurs humains dans une perspective de conception centrée utilisateur (Bastien \& Scapin, 2004): " une phase d'analyse des besoins impliquant les futurs utilisateurs, une phase de conception, de test et de réalisation et, une phase d'implémentation et de suivi » (pp. 451452). Au final, la démarche mise en œuvre déborde ce positionnement initialement prévu pour une position qui se situe à l'interface entre la MOA et la MOe, représentées respectivement par le partenaire industriel, sa filiale, et l'équipe de recherche pilotant le projet. L'équipe d'ergonomes participe à ce projet avec en ligne de mire la transformation du travail et le développement des compétences professionnelles, dans un double objectif de performance et de santé. La première étape de l'analyse de la demande visait alors à construire ce positionnement ce qui nécessitait notamment d'impliquer différents acteurs de l'entreprise au-delà de ceux initialement identifiés (le directeur du centre de formation, et le référent du service de réalité virtuelle) : les formateurs, enseignants, formés, le service de prévention (IPRP, Médecin du travail), l'ergonome interne, les compagnons, chefs d'équipes $(\mathrm{N}+1)$, chefs de ligne $(\mathrm{N}+2)$ et le CHSCT.

La compréhension du projet et l'analyse de la demande se sont ainsi échelonnées sur une période d'un an et trois mois : des premiers contacts avec le partenaire industriel et sa filiale, initialement circonscrits aux possibilités d'accès au terrain d'investigation, jusqu'à la présentation de l'étude et sa validation devant le Comité Hygiène, Sécurité et Conditions de Travail du site (CHSCT). Cette phase a permis de redimensionner le projet scientifique en réinterrogeant les «intentions » du projet (Barcellini, Van Belleghem, \& Daniellou, 2013) par la mise en perspective des connaissances développées sur le travail réel et des possibilités technologiques ; mais aussi au regard de la pertinence, du point de vue du travail, des options scientifiques jusqu'ici retenues. Il s'est traduit par le passage d'un objectif initial de formation aux «gestes professionnels» par la Réalité Virtuelle dans une formation conçue autour du futur EVF prenant totalement en charge le formé ; au projet de conception d'un EVF participant, dans le cadre d'une formation plus large, au développement de l'organisation cognitive de l'action lors de la réalisation de tâches de montage-assemblage (Boccara \& Delgoulet, 2013).

10 Nous tenons ici à préciser que nous n'avons pas participé au montage du projet, nous l'avons intégré au cours de la première année. Ce paragraphe reflète ainsi notre compréhension, de l'organisation initiale du projet, élaborée à partir de la lecture du document de réponse à l'appel à projet et des premiers échanges que nous avons eus avec les référents des différentes équipes partenaires à notre arrivée dans le dispositif. 


\section{2.- Le travail des monteurs-assembleurs de l'aéronautique}

\subsection{1.- L'organisation du travail}

L'organisation du travail est de type « lean manufacturing ». La production se fait en petites, voire très petites séries, de produits. Chaque avion étant destiné à une compagnie aérienne particulière, de nombreuses variantes existent dans le montage des pièces en fonction des options retenues par le client. Les éléments d'avion passent de poste en poste dans les ateliers sur une ligne flexible d'assemblage avant d'être livrés. Les cadences sont dépendantes du type d'avion et notamment de la maitrise du processus de fabrication. Les cadences les plus «faibles » se trouvent au poste où sont assemblés les avions les plus récents; elles sont alors de l'ordre de deux avions par mois (premier poste étudié). Les cadences les plus «élevées »s'observent sur les lignes de fabrication les plus anciennes; elles sont de l'ordre de deux jours. Des cadences intermédiaires existent également, elles sont de l'ordre de trois avions par semaine (second poste étudié).

L'organisation temporelle des équipes prend la forme de l'alternance $(2 * 8)$ avec une période de recouvrement réduite à six minutes entre l'équipe du matin (5h-13h36) et celle de l'aprèsmidi (13h30-22h) et une rotation hebdomadaire des équipes entre les deux horaires. Un agent de maitrise veille à la coordination du travail dans chaque équipe. Il est le garant du respect des règles, procédures et consignes de travail dans une triple visée d'assurance de la qualité, des coûts et des délais.

La répartition du travail au sein d'une équipe se fait lors de la prise de poste, à $5 \mathrm{~h}$ ou à 13h30. Un compagnon effectue de préférence une opération dans sa totalité pour des questions de responsabilité et de traçabilité du travail réalisé : celui qui a fait des erreurs devra ensuite faire les réparations. Toutefois, compte tenu de la durée de réalisation des opérations (parfois plusieurs heures), les compagnons sont souvent amenés à travailler à plusieurs sur une même opération ou en se répartissant des sous-opérations lorsque ceci est matériellement et temporellement possible.

\subsection{2.- Les tâches des compagnons aux postes de fabrication}

Le travail des compagnons est classiquement régi par des consignes (d'utilisation des outils, de sécurité, de qualité), des règles de métiers, des procédures, matérialisées notamment dans les plans de construction de chaque élément d'avion et les gammes de travail (pièces, fixations, ordonnancement des opérations, documents règlementaires à consulter et temps imparti). Au quotidien les compagnons travaillent peu avec les plans sous les yeux. Avec l'expérience, ils ont mémorisé les opérations à réaliser ainsi que leur ordre de réalisation. Toutefois, les plans constituent une ressource importante en cas de doute ou pour les compagnons novices sur le poste. À titre d' exemple, le poste de «barque » observé nécessite selon les compagnons qui y travaillent environ deux ans pour être maîtrisé par un compagnon expérimenté dans le métier.

Les tâches prescrites aux deux postes étudiés sont définies comme relevant de la phase de « finition ». Elles consistent à installer différents supports fixes et pièces d'aménagement de l'avion qui viendront par exemple guider le passage de câbles électriques ou de tuyauteries permettant d'alimenter l'avion et de le viabiliser. Ainsi, cinq opérations sont programmées au poste de barque observé qui sont détaillées en 49 sous-opérations pour la partie haute de cet élément (l'intérieur du fuselage). Leur temps de réalisation est estimé à 128 heures de travail, avec un passage de l'élément sur poste compris entre deux et trois jours. Au total 314 pièces sont ainsi fixées sur chaque barque par une équipe de quatre compagnons.

Les compagnons doivent réaliser l'assemblage de pièces aéronautiques au moyen d'un ensemble de fixations principalement constitué de trois grands types de rivets et de vis. L'analyse des tâches prescrites et effectives permet de repérer dix-huit tâches élémentaires, omniprésentes dans les opérations réalisées par les compagnons formant ainsi la base du métier : aléser, coller, contre-percer, dérocher, ébavurer, agrafer, fraiser, moucher, percer, 
renvoyer, etc. Autrement dit, elles constituent une base minimale de «ce qu'il faut maîtriser et savoir réaliser» pour assembler des pièces d'avion. Selon les fixations à poser et les conditions d'assemblage, elles sont, peuvent ou doivent être ordonnées différemment. À chacune de ces tâches correspondent des normes de tolérance de l'ordre du dixième de millimètre, voire au centième pour certaines. L'activité des compagnons est ainsi d'une extrême précision.

Par ailleurs, la réalisation de ces tâches s'effectue au moyen d'un ensemble très varié d'outils et de matériels spécifiques au métier (des dizaines, voire centaines). À titre d' exemple, nous avons inventorié un minimum de 45 outils qui doivent être mobilisés et maîtrisés pour réaliser les tâches «de base »: perceuse, pistolet à riveter, visseuse, bombonne LGP, foret (avec ou sans renvoi d'angle), aléseur, pince à agrafes, etc.

\subsection{3.- Une grande diversité des situations de travail}

Le travail des compagnons est réalisé dans une grande diversité de situations, compte tenu des exigences de fabrication en petites séries sur plusieurs modèles d'avion, et une variabilité dans la configuration de ces situations au cours du temps. Les observations et entretiens exploratoires menés auprès des compagnons et de leurs encadrants de proximité permettent de pointer les principaux éléments de diversité et variabilité de ces situations. Sept sont ici répertoriés :

— des niveaux d'ambiance physique (sonore, éclairement, thermique) qui fluctuent selon les tâches réalisées par un compagnon ou ses collègues (les tâches de rivetage étant particulièrement bruyantes, par exemple), selon les phases de travail ou la période de l'année (fluctuation de la température ambiante et des sources de lumière entre l'hiver et l'été) ;

— des pièces à monter/assembler qui se distinguent par la taille, l'épaisseur et le poids ; d'après nos observations, elles sont le plus souvent manipulées par un seul compagnon, excepté lorsqu'elles ont été préparées à l'établi en sous-ensemble pouvant faire plusieurs mètres ;

— des outils qui se différencient par leur poids (léger/lourd), leur fonctionnement (manuel/semi-automatique/automatique, etc.), leur état (degré d'usure, état de propreté), ce qui suppose des stratégies et des modes opératoires différents: par exemple, l'utilisation d'un foret usagé nécessite que l'on exerce une force plus importante sur la perceuse et/ou une vitesse de rotation plus rapide du foret que lorsque le foret est neuf ;

— des éléments d'avion qui font entre $9 \mathrm{~m}^{2}$ et $15 \mathrm{~m}^{2}$, sur lesquels on peut compter jusqu'à quatre compagnons y travaillant simultanément : ceci suppose que chacun sache ce qu'il a à faire, ait organisé sa zone d'intervention de manière à ne pas déranger le travail des autres et ainsi limiter les va-et-vient jusqu'à l'établi ou aux espaces de rangement et de stockage des outils et des pièces. Ceci suppose d'avoir conscience de l'évolution du travail des autres et de l'aménagement provisoire de leur zone propre d'intervention ;

— des configurations d'espace de travail et d'accessibilité des zones d'assemblage qui obligent les compagnons à changer très fréquemment de postures, donnant ainsi à voir un enchaînement de postures «atypiques» plus ou moins pénalisantes pour la santé : rivetage assis sur une pièce, la tête penchée vers le bas ; perçage ou rivetage debout-plié, la tête penchée vers le bas; perçage en équilibre sur un marchepied avec les bras levés au-dessus des épaules; perçage à genoux, penché en avant, les bras enfoncés dans les interstices de la structure du plancher; perçage et rivetage allongé sur des traverses métalliques, etc.

— des situations de rivetage à deux qui nécessitent de partager un espace réduit conduisant dans la plupart des cas à un contact physique entre compagnons : accroupis devant le rivet à écraser les épaules se touchant ou, allongés l'un en face de l'autre, les bras tendus vers le lieu du rivetage et aussi de part et d'autre d'une paroi. Cette proximité dans la 
réalisation du travail met parfois les compagnons dans une situation paradoxale : ils pourraient a priori voir ce qu'ils font, pourtant leur proximité physique pendant la réalisation de la tâche peut masquer leur propre travail ainsi que celui de l'autre ;

— des consignes, procédures et règles (opérations, qualité, sécurité) plus ou moins applicables selon les situations de travail; le port de certains EPI ${ }^{11}$ est ici exemplaire, il n'est pas possible de porter une casquette lorsque l'on travaille la tête inclinée ou en bas, ou encore en ayant un contact physique lors des phases de travail à deux, ce qui arrive régulièrement aux deux postes observés.

\subsection{4.- Exigence de qualité et réparation des défauts}

Comme déjà souligné, l'assemblage est un travail d'une extrême précision qui suppose une combinaison dans l'action de nombreuses dimensions de l'activité physique et cognitive. Compte tenu des exigences de production, les défauts ne sont pas rares. Une partie des défauts supposent que les services de contrôle de la qualité produisent des dérogations et des procédures de réparation ad hoc qui ne compromettent pas la qualité de l'assemblage final. Dix catégories de défauts ont été définies par l'entreprise : perçage hors tolérance, coups, rayures et déformations du fuselage, perçage parasite d'une pièce, etc. Trois niveaux de dérogation existent : plus la réparation est de niveau élevé, plus son coût l'est également du fait de la mobilisation du bureau d'étude et du temps supplémentaire induit par la réparation.

Au poste de finition de la barque, 12930 défauts ont ainsi été identifiés sur une période de quatre mois : $75 \%$ d'entre eux ont fait l'objet d'une dérogation. Les défauts les plus répandus sont ceux de «perçages hors tolérances » $(43,3 \%)$. On constate également que les « coups, rayures et déformations » représentent une part non négligeable $(31,7 \%)$; elles ne sont pas directement liées à la réalisation des tâches élémentaires du métier, mais à la manipulation des outils, outillages et aux déplacements des compagnons sur l'élément d'avion. Viennent en troisième, les «perçages parasites » (19,3\%), c'est-à-dire des perçages malencontreux de pièces situées en arrière plan de celle à percer. Les chefs de ligne associent ces derniers défauts au travail des nouveaux recrutés qui ne vérifieraient pas la configuration de leur champ de perçage avant de s'engager dans la tâche. Ils les présentent comme ayant de forts enjeux financiers dus au temps de dépose de la pièce indument percée, à sa mise au rebus, à la commande et au montage d'une nouvelle pièce, voire à la réparation des pièces endommagées.

\subsection{5.- Les accidents du travail des nouveaux recrutés}

En collaboration avec le service de prévention, nous avons travaillé sur la base de données des accidents du travail ayant eu lieu sur ce site durant six années précédant notre étude (2006-2011). Au total, cette base contient 339 événements, soit une cinquantaine d'événements répertoriés par an.

Afin de travailler la phase de transition entre formation et travail productif, l'analyse des accidents, se centre sur la seule population des compagnons récemment recrutés (entre 0 et 2 ans d'ancienneté, ayant suivi auparavant une formation dispensée au centre de formation). Elle permet de dégager quelques tendances de l'accidentologie des novices qui sont pour la plupart des salariés de moins de 30 ans. Parmi l'ensemble des accidents, $30 \%(102 / 339)$ concerne les novices. Ils se répartissent comme suit: $52 \%$ relève d'une manipulation d'outils (perceuse, pince à agrafes) ou de matériel professionnel (53/102), $24 \%$ est lié à des chutes (24/102), $14 \%$ correspond à des chocs contre des éléments d'avion ou des bâtis et, $10 \%$ à des douleurs soudaines (maux de dos principalement).

Les blessures par manipulation d'outils ou matériels (les plus fréquentes) peuvent avoir lieu lorsqu'un compagnon réalise seul une tâche : principalement à l'occasion de la préparation du matériel ou des outils, de la maintenance des outils ou de la manipulation des agrafes qui

11 Équipements de Protection Individuelle. 
permettent d'assembler momentément plusieurs pièces avant de les fixer définitivement avec des vis ou des rivets. Elles peuvent survenir à l'occasion d'un travail à deux (un compagnon blessant son partenaire, novice), dans le cadre de la réalisation du rivetage essentiellement. Les membres touchés sont les doigts (percés, écrasés, pincés, écorchés); le visage (projection d'agrafes, choc contre des éléments d'avion, projection de copeaux métalliques dans les yeux) ; les chevilles (entorses lors de chutes); le dos (douleurs survenant lors d'un port de charge, d'un changement de posture, d'un travail en force).

\section{3.- Le travail en formation : l'activité formateurs-formés}

\subsection{1.- L'organisation de la formation}

Rappelons que la formation professionnelle destinée aux intérimaires se déroule sur le site de formation. Chaque session accueille un groupe de 12 formés qui valident leurs apprentissages par l'obtention d'un CQPM. La formation est coordonnée et animée par un des deux formateurs présentés précédemment. Chaque groupe est pris en charge par un formateur.

Deux semaines de cours magistraux (CM) en salle sont dévolues aux contenus technicoréglementaires organisés en 17 modules thématiques. L'analyse documentaire, complétée d'entretiens avec les formateurs et les formés, souligne l'ampleur des connaissances que les formés doivent acquérir pendant ces cours. Elles sont relatives aux pièces, outils, outillages, fixations et procédés à utiliser, spécifiques à l'aéronautique. La présentation du contenu en modules accolés, mais disjoints (caractère renforcé par la succession de nombreux intervenants), laisse toutefois aux formés le travail d'une part, de construction des liens entre les noms des objets, les outils, les tâches à réaliser et les procédés et, d'autre part, de représentation de la dynamique de l'activité des assembleurs-monteurs. Bien que les formateurs tentent de rendre compte de ces liens et de les expliciter, un ensemble de facteurs organisationnels contraint leur activité en formation : la séparation des deux espaces celui de la ligne-atelier et celui de la salle de cours (difficulté de faire des allers-retours entre ces deux espaces pour illustrer ce qui est abordé en $\mathrm{CM}$ ou revenir sur des concepts et phénomènes physiques sous-jacents lorsque les formés sont en TP), l'organisation de la salle de cours en rangs de tables, les supports de cours statiques (diaporamas, documents papier) et la durée courte par rapport au volume des contenus à apprendre.

Six semaines sont ensuite dédiées à des travaux pratiques (TP) au travers de trois exercices réalisés sur la ligne-atelier, sous la tutelle d'un formateur. Ces exercices sont accomplis sur des pièces avioniques de tailles réduites par rapport aux pièces en production. La ligneatelier est composée d'un grand établi, comprenant douze postes de travail, sur lequel est réalisé l'exercice 1 et, trois types de bâtis verticaux (dix bâtis au total) pour la réalisation des deux autres exercices de la formation. Les formés travaillent principalement dans des positions debout, à genou ou sur un marchepied face à la zone de travail. Deux groupes de formés peuvent être en parallèle sur la ligne-atelier.

Le passage des CM aux TP est considéré comme une période critique de la formation tant par les formés que par les deux formateurs. Les formés interviewés disent se sentir «perdus» pendant les deux premières semaines sur la ligne-atelier. Cette période correspond au temps de réalisation du premier exercice pratique, qui met en scène la majorité des outils, des fixations et des tâches élémentaires. Les verbatim suivants, issus de quatre entretiens avec des formés, fournissent un aperçu des obstacles auxquels ceux-ci se confrontent :

Formé 1 : «J'étais juste perdu un petit peu au niveau des outils, des appellations, donc les différentes fraises, les fraises monoblocs tout ça, donc c'est pour les appellations ».

Formé 2: "Pour ma part, j'ai eu tendance au départ à mélanger certaines choses avec certaines choses, puisqu'il fallait que je reprenne le cahier pour voir... selon les démarches, 
ou alors selon les produits qu'on utilise, pour dégraisser ou alors pour coller, ou alors... et nous on s'en souvient, mais des fois, on mélange plus ou moins selon, soit les couleurs de produits ou autres... ».

Formé 3 : «Pendant les quinze jours [des CM] on ne savait pas de quoi il [le formateur] parlait [...]. Mais je ne peux pas assimiler un mot avec un objet qu'on ne connaît pas pendant que... Ce n'est pas évident. [...] Il [le formateur] parlait de 'fraise' ou de 'tas'... un peu de tout quoi, les produits qu'on utilise. Nous c'est des mots nouveaux, on ne connaît pas le produit, on ne connaît pas l'outil, donc après on était en atelier, il nous disait d'aller chercher tel outil, il nous en avait parlé, mais on ne savait pas quoi attraper ».

Formé 4: "Quand il [le formateur] nous parlait en cours, on va dire... on ne voit pas vraiment, on voit sur l'image [photo ou dessin des outils et du matériel présenté en cours], mais on ne sait pas vraiment ce que c'est, et ce que ça fait. Donc une fois qu'on arrive à l'atelier, au début on a un petit peu de mal, mais on s'y fait assez vite. En même temps, on le fait tous les jours...».

Ainsi, les liens laissés à la charge des formés pendant les CM deviendraient un objet d'apprentissage au cours de la réalisation de ce premier TP. Plus spécifiquement, les formés déclarent maîtriser les liens entre le lexique professionnel, les outils, les buts à atteindre ainsi que l'ordre temporel des opérations à la fin de cet exercice. Ils ont construit durant cette période le «ce qu'il y a à faire » en interaction avec le «comment faire » (quelles ressources mobilisées), construction qui se poursuivra lors des deux autres exercices de la formation. La sensation «d'être perdu » des formés dans les premières semaines d'atelier proviendrait alors de leurs difficultés à savoir «ce qu'il y a à faire » jouant un rôle d'orientation dans la construction du «comment faire». Autrement dit, ils ont des difficultés à identifier les dimensions explicites et implicites de la tâche à réaliser, bien qu'une fiche technique comprenant des plans et des explications détaillés ait été construite pour guider les formés dans la réalisation de l'exercice.

\subsection{2.- La dimension didactique du travail sur la ligne-atelier}

Des observations menées lors des sessions sur la ligne-atelier, retenons que l'activité didactique des formateurs renvoie à l'organisation des séances et au «pilotage » des formés lors de la réalisation des exercices. Le contenu des apports en CM et des TP ayant été construit et validé par la branche professionnelle qui délivre le CQP (la Métallurgie), les formateurs ont peu de latitude sur ce volet. En revanche, ils doivent en permanence veiller à la disponibilité des lieux, aux possibilités d'articulation éventuelle de deux groupes formés en parallèle, l'ordonnancement des séances, la disponibilité des intervenants issus des services en production (qui peuvent déplacer ou annuler leur intervention à tout moment, les contraintes de production primant sur celles de la formation), ceci dans le respect d'une progression qui a du sens pour les apprenants. Le pilotage comporte quant à lui un double rôle des formateurs: ils sont tour à tour, ou conjointement, «accompagnateurs » et « évaluateurs » du travail produit. En tant qu'accompagnateurs, ils orientent (« ce qu'il y a à faire ») et étayent le travail d'apprentissage des formés à partir d'un ensemble de savoirs techniques, réglementaires et de métier («comment faire»). En tant qu'évaluateurs, ils contrôlent les acquis des formés. Ces deux rôles offrent aux formateurs des occasions de transposer en formation des éléments critiques des situations de production en tenant momentanément le rôle d'un supérieur hiérarchique, d'un contrôleur qualité ou d'un autre compagnon, ce qui pourrait s'apparenter à de la simulation langagière (Nicolas, 2000a ; 2000b ; Béguin \& Weil-Fassina, 1997) à visée didactique. Ils jouent également sur les facteurs organisationnels: manque d'outils, leur usure, leur mauvais rangement, les dimensions collectives du travail (dépendance des tâches entre les compagnons, partage des espaces et des outils, etc.) ou l'encombrement des postes et leurs effets sur le travail. Ces simulations peuvent aussi bien être organisées qu'opportunistes en fonction des situations de formation et, des erreurs ou des interventions des formés. Elles constituent alors des opportunités d'apprentissage de dimensions du travail que la ligne-atelier, en tant que 
dispositif de formation, ne porte pas intrinsèquement, mais que les formateurs, anciens compagnons dans les ateliers de production, peuvent introduire grâce à l'expérience qu'ils en ont.

Dans une autre perspective (Rogalski, 2003), les interventions des formateurs agissent et/ou réagissent à la dynamique du développement des compétences des formés. Leurs interventions se basent donc pour partie sur le diagnostic qu'ils font de l'état et des évolutions possibles des compétences des formés, ainsi que sur les effets anticipés de leurs interventions sur ce développement. Comme observé dans d'autres travaux (Antolin-Glenn, 2005 ; Boccara, 2011 ; Vidal-Gomel, 2001), les interventions des formateurs peuvent être caractérisées par le mode (à la cantonade, au groupe, à un seul formé, etc.), la forme (explication, correction, guidage, etc.) et, le contenu (buts, connaissances, règles, procédures, etc. ; Boccara, 2011) et leur(s) registre(s) du métier (réglementaire, technique, sécuritaire, éthique, etc.). Au cours des entretiens, les formateurs soulignent l'importance de s'adresser à l'ensemble du groupe, afin d'éviter les répétitions et d'assurer une homogénéité des explications fournies. Ils considèrent également que la démonstration est un moyen favorable à la compréhension des formés, notamment pour illustrer et expliquer les indicateurs difficilement verbalisables comme le bruit, le temps ou les sensations tactiles. Les démonstrations sont ainsi utilisées pour « faire voir aux formés ce qui ne se voit pas » quand on n'est pas du métier. De manière complémentaire, les formateurs «tournent» en permanence dans l'atelier et interviennent de manière opportuniste auprès des formés en fonction des difficultés ou des erreurs qu'ils observent in situ. Cette stratégie des formateurs permet également aux formés de les solliciter facilement au cours de la réalisation des exercices. De cette manière, les formateurs ont un suivi aussi bien collectif qu'individuel de leurs stagiaires.

À partir du dispositif de co-construction (cf. phase 3 de la démarche) et des travaux menés précédemment dans ce secteur professionnel (Aubert-Blanc, 2009), nous avons reconstitué deux structures conceptuelles (Pastré, 1999; 2011): l'une sous-jacente aux tâches de perçage, contre-perçage, renvoi d'un trou et alésage; l'autre sous-jacente à la tâche de rivetage seul ou à deux. Nous avons ainsi mis en évidence trois concepts pragmatiques: $1)$ «l'équerrage ${ }^{12}$ » et «l'avance ${ }^{13}$ » dans la première structure conceptuelle ; 2) «l'équerrage » et «l'écrasement ${ }^{14}$ » pour la seconde. Ces structures conceptuelles ont fait l'objet de discussion avec les deux formateurs qui les ont enrichies avant d'être validées. Dès le premier exercice de la formation, les formés sont amenés à mobiliser et construire progressivement ces concepts par la réalisation de l'action et les interventions des formateurs. En effet, les interventions des formateurs portent dès les premières heures de formation dans l'atelier sur les concepts identifiés (e.g. équerrage, avance, etc.), des paramètres (e.g. vitesse de rotation, accessibilité du trou, etc.) ou encore des indicateurs (e.g. l'épaisseur, la matière des pièces ou bien le graissage, l'usure, le diamètre du foret utilisé). Ces éléments ne sont pas abordés par les formateurs les uns après les autres sans lien explicite entre eux. Au contraire, les interventions des formateurs permettent de préciser aux formés les relations entre ces différents éléments, nécessaires pour identifier l'état de la situation et les opérations à réaliser. Elles mettent à plat les « liens » que les formés doivent

12 Le concept pragmatique d'« équerrage » renvoie au concept scientifique de perpendicularité. Il agrège un ensemble de paramètres des situations de travail en vue de réaliser des trous orientés « correctement » en $\mathrm{x}, \mathrm{y}$ et $\mathrm{z}$ et cela en fonction de la forme et de l'épaisseur des pièces perforées.

13 Le concept pragmatique d' '« avance » renvoie à la progression du foret dans la matière qui dépend notamment de la force appliquée sur le bloc moteur de la perceuse par la personne, la vitesse de rotation du foret, son diamètre, son tranchant, les qualités de sa matière, mais aussi celles de la pièce à percer ou contre-percer et son épaisseur.

14 Le concept pragmatique d' '« écrasement » renvoie à la compression progressive des rivets qui dépend notamment des caractéristiques du rivet (forme, diamètre, longueur, matière, etc.), de la frappe sur la tête du rivet, (type de pistolet et de bouterolle à rivet, force, fréquence et durée) et de « tas » (taille, forme, matière) utilisée en contre-point de la frappe pour faciliter l'écrasement du rivet (épaisseur, matière, force exercée). 
construire pour comprendre les situations auxquelles ils font face et savoir ce qu'ils doivent réaliser. Toutefois, bien que ces concepts sont l'objet des interventions des formateurs, ils ne semblent pas être l'objet d'une transposition formalisée et structurée pour favoriser leur développement par les formés au cours de la formation. Les structures conceptuelles mises au jour sont alors susceptibles de constituer des futurs outils pour l'ingénierie de formation : évaluation des exercices et de la progression existante, mais aussi création de nouveaux exercices pour les futures situations de formations.

\section{4.- Discussion et Conclusion}

Cette étude visant à instruire les situations de travail existantes (en production et formation) s'inscrit donc dans une perspective d'intervention en ergonomie où concevoir des artefacts n'est pas uniquement proposer des critères de conception ou d'évaluation « ergonomique ». C'est aussi donner des occasions de développement aux acteurs impliqués dans la conception (Rabardel, 1995), c'est-à-dire ici à ceux rencontrés en formation (apprenants ou formateurs), en production (ergonome, préventeurs, ou compagnons), mais aussi en conception (informaticiens, industriels et designers, partenaires du projet). Comme le soutiennent Béguin et Cerf (2004), la conception peut avoir plusieurs horizons, dont celui de concevoir pour des genèses. Cet horizon de la conception dépasse alors la fin du processus formel de conception (le projet). Il invite à anticiper les possibilités d'appropriation et d'usages pouvant dans certains cas amener des transformations ou des reconfigurations de l'artefact. Ainsi, il introduit une réflexion pendant le projet sur les frontières du système, les marges de manœuvre qu'ils offrent, voire celles dont il pourrait favoriser le développement.

Au-delà de notre contribution, par l'analyse de l'existant, à la conception d'un prototype d'EVF dans le cadre d'un projet de recherche, l'analyse des travails permet de tenir les enjeux des différents partenaires scientifiques et industriels de ce projet ainsi que ceux portés par les acteurs de terrain. Parce qu'au cours de cette démarche, la focale de l'analyse, les acteurs impliqués ainsi que les résultats produits évoluent, se constitue un processus de conception dialogique qui favorise et oblige à la construction de compromis. Dans cette visée développementale (Béguin, 2007), on trouve par exemple des arbitrages entre les souhaits des futurs utilisateurs (direction, formateurs ou formés) et les possibilités technologiques actuelles; ou encore, entre les scénarios techniquement possibles, porteurs de nouveaux défis scientifiques pour des chercheurs-concepteurs, et ceux souhaitables du point de vue du développement des compétences, de la préservation de la santé des futurs compagnons ou encore des marges de manœuvre des formateurs, et par là-même de la performance du système. Concevoir des artefacts pour la formation dans le cadre d'une analyse des travails est alors aussi l'occasion de requestionner le travail productif et les acteurs qui l'organisent (dans ses choix organisationnels ou ses outils de suivi de la prévention des risques pour ne donner que deux exemples). En cela nous nous situons dans la lignée des travaux d'ergonomes visant, au travers de la formation, la transformation du travail (Falzon \& Teiger, 2011 ; Lacomblez, Bellemare, Chatigny, Delgoulet, Re, Truel, \& Vasconcelos, 2007). Le périmètre de la recherche-intervention était à l'origine circonscrit à des situations existantes de travail pour la conception de l'EVF. Un des enjeux de la démarche d'analyse «des travails » a été d'étendre ce périmètre en impliquant plus largement les acteurs du site de production (prévention, médecine du travail, ergonomie et production) et ceux de la formation (formateurs et formés). N'étant pas missionnés pour transformer les situations de travail (en production ou formation), cet élargissement visait à intégrer ces acteurs afin qu'ils contribuent en interne à l'amélioration des situations de travail à partir des analyses produites au cours du projet. Cet élargissement s'est appuyé sur une analyse de la demande intégrée à une analyse du projet. Ceci se justifie d'une part du fait du format des réponses à ce type de projets (découpage en tâches et sous-tâches, centration sur les connaissances scientifiques actuelles ou à développer et leurs domaines d'application) 
et des contraintes temporelles fixées par le financeur (réponse à transmettre quelques mois après la diffusion de l'appel) qui rend difficile l'implantation dès la réponse d'une démarche réellement coordonnée entre industriels et équipes de recherche et entre les équipes de recherche elles-mêmes. D'autre part, notre absence lors du montage et du démarrage du projet a sans doute contribué au besoin de clarification du projet à notre arrivée. Rétrospectivement, cette phase comporte des enjeux stratégiques sur la place des différents acteurs, les structures de discussion/dialogue et prise de décisions, le périmètre, l'orientation, l'histoire du projet qui inciterait à généraliser ce type d'analyse comme une phase à part entière de la recherche-intervention. C'est notamment cette phase qui a permis l'implication des formateurs dans le dispositif d'analyse des situations de formation, ce qui a contribué à la mise au jour de transformations souhaitables de leur situation de travail (aménagement des locaux de formations; amélioration des outils pédagogiques) et, à une réflexion sur leurs propres pratiques de formation favorable à leur développement et à la préservation de leur marge de manœuvre.

Plus concrètement, la démarche d'analyse des travails propose de produire et d'alimenter un référentiel de situations (Boccara, et al., 2014 ; Mayen, et al., 2010) synthétisant des repères pour la conception jouant le rôle d'objet intermédiaire dans le processus de conception (Jeantet, 1998 ; Vinck, 2009). Ce référentiel de situations de formation intégrant : 1) des facteurs organisationnels et situationnels en production et en formation, 2) des sources potentielles d'accidents et de défauts en production, 3) les tâches, les procédés, les règles et les outils et, 4) des éléments concernant la conceptualisation pragmatique et des organisateurs de l'activité des compagnons. Ce référentiel a été conçu avec le double objectif d'un outil opérationnel pour : 1) les responsables de l'ingénierie de la formation (formateurs, professeurs et directeur du centre de formation) ; 2) les concepteurs de l'EVF en vue de proposer des situations potentielles de développement dans et avec le futur EVF (Mayen, 1999).

Du point de vue de l'ingénierie de la formation actuelle, les films réalisés avec les deux formateurs alimentent les $\mathrm{CM}$ en offrant la possibilité de mettre en mouvement et de rendre compte de la dynamique du travail de montage-assemblage jusqu'ici traitée de manière exclusivement statique; forme qui ne satisfaisait ni les formateurs, ni les formés (cf. plus haut). Le référentiel de situations a quant à lui retenu l'intérêt des différents acteurs. Il suppose toutefois un travail d'appropriation qui n'a pas encore été mené à ce jour par les acteurs de l'ingénierie de formation. Les conditions de cette appropriation et les démarches d'accompagnement envisageables constituent sans doute un champ de recherche à investiguer pour renforcer l'action de l'ergonome en conception de formation.

Du point de vue de la conception de l'EVF, le référentiel de situations a été une ressource et un outil pour définir et construire l'interface matérielle (commandes, dispositif technique, etc.), l'interface logiciel avec un ensemble de scénarii de formation, mobilisant potentiellement des agents virtuels et répondant à différents modes d'utilisation selon le type de situations réelles de formation envisagé (en salle vs. en atelier, seul vs. en groupe, avec un formateur vs. sans formateur, etc.). Le référentiel proposé offre ainsi un ensemble d'éléments pouvant être utilisés et combinés de manière variée, afin de simuler des situations de formation en fonction des questions posées au cours du processus de conception et de leurs évolutions. Il participe également à guider l'analyse fonctionnelle et l'élaboration des spécifications avec et pour l'équipe de conception en prenant en compte conjointement l'utilité, l'utilisabilité et la dimension apprentissage du futur système (Delgoulet \& Boccara, 2013).

En conclusion, l'analyse des travails ouvre des perspectives pour la recherche et l'intervention dans le champ de la formation professionnelle et, plus particulièrement, pour la conception de dispositifs, d'outils et/ou de contenu de formation. L'exemple pris ici souligne le potentiel de re-interrogation en vue de transformation des situations de travail en 
production et en formation en entrant par des demandes de formation, d'une part ; en vue de la précision et la réorientation des intentions du projet de conception, d'autre part. Enfin, elle contribue à la réflexion sur les multiples objets et finalités des processus de conception pour dépasser les approches centrées sur l'élaboration et le respect de critères « ergonomiques ».

\section{REMERCIEMENTS}

Ce travail a reçu de soutien financier de l'Agence Nationale de la Recherche (ANR-10-CORD-0014). Nous remercions par ailleurs vivement les formateurs et l'ensemble des participants sur les sites de production et de formation, sans qui ce travail n'aurait pas été possible.

\section{BIBLIOGRAPHIE}

Amigues, R. (2003). Pour une approche ergonomique de l'activité enseignante. Skholê, hors-série 1, 516.

Amokrane, K., Andriot, C., Boccara, V., Carpentier, K., Convard, T., Delgoulet, C., Guerriero, E., Lourdeaux, D., Vinchon, G., \& Wagrez, K. (2013). Des STIC et des hommes. Nikita: Natural Interactions, Knowledge, Immersive system for Training in Aeronautic. Les rencontres du numérique de l'ANR, les 17 et 18 avril, Paris.

Anastassova, M., \& Burkardt, J.-M. (2009). Automotive technicians' training as communitu-ofpractice: implementation for the design of an augmented reality teaching aid. Applied ergonomics, 40, 713-721.

Anastassova, M., Burkhardt, J.-M., Mégard, C., \& Ehanno, P. (2005). Results from a usercentred critical incidents study for guiding future implementation of augmented reality in automotive maintenance. International Journal of Industrial Ergonomics, 35(1), 81-91.

Anastassova, M., Burkhardt, J.-M., Mégard, C., \& Ehanno, P. (2007). L'ergonomie de la réalité augmentée pour l'apprentissage. Le Travail Humain, 70(2), 97-125

Antolin-Glenn, P. (2005). Le développement des compétences à la coopération dans la formation à la prise de décision distribuée hiérarchisée. Le cas de la formation continue à la gestion opérationnelle des officiers sapeurs pompiers. Thèse de Doctorat, Université Paris 8, Saint-Denis.

Arborio, A.-M., \& Fournier, P. (1999). L'enquête et ses méthodes : l'observation directe. Paris: Nathan Université.

Aubert-Blanc, S. (2009). Apport de l'ergonomie à la définition du contenu des savoir-faire de métier et à l'organisation de leur transmission. «C'est pas compliqué de percer un trou ! ». Séminaire «Transmission des savoirs et mutualisation des pratiques en situation de travail » (pp. 33-41). Rapport de recherche du CEE.

Barcellini, F., Van Belleghem, L., \& Daniellou, F. (2013). Les projets de conceptions comme opportunité de développement des activités. In, P., Falzon (Ed.), Ergonomie constructive (pp. 191206), Paris: PUF.

Bastien C., \& Scapin, D. (2004). La conception de logiciels interactifs centrés sur l'utilisateur : étapes et méthodes. In, P. Falzon (Ed.), Ergonomie (pp. 451-462), Paris: PUF.

Beaujouan, J., Aubert, S. \& Coutarel, F. (2013). Formateur-animateur : un rôle sous-estimé et à outiller dans la conception de dispositifs de formation. Cas de la formation de dispositifs de formation. Cas de la formation aux opérations de contre-perçage sur avion. Journées Scientifiques de Nantes, 5-7 juin 2013. https://hal.archives-ouvertes.fr/hal-00973460.

Béguin, P. (2007). Prendre en compte l'activité de travail pour concevoir. Activités, 4(2), 107-114.

Béguin, P., \& Cerf, M. (2004). Formes et enjeux de l'analyse de l'activité pour la conception des systèmes de travail. Activités, 1(1), 54-71.

Béguin, P., \& Weil-Fassina, A. (1997). La simulation en ergonomie : connaître, agir et interagir. Toulouse: Octarès.

Berglund, M., \& Karltun, A. (2012). Towards understanding and managing the learning process in mail sorting. Work, 41(2), 115-126.

Berthelette, D. (1995). L'évaluation de programmes de formation en ergonomie. Éducation permanente, 124(3), 97-109.

Blanchet, A., \& Gotman, A. (1992). L'enquête et ses méthodes : l'entretien. Paris: Nathan. 
Boccara, V. (2011). Développement des compétences en situation de tutelle au cours de la formation à la conduite automobile. Apports croisés de la psychologie ergonomique et de la psychologie sociale. Thèse de Doctorat, Université Paris 8, St-Denis.

Boccara, V., \& Delgoulet, C. (2013). Articuler les démarches d'analyse du travail en ergonomie et en didactique professionnelle pour la conception d'un EVAH. Journées Scientifiques de Nantes, 5-7 juin 2013.

Boccara, V., Vidal-Gomel, C., Rogalski, J., \& Delhomme, P. (2014). Concevoir des référentiels comme des outils pour les formateurs ? Réflexions à partir de la formation initiale à la conduite automobile. In B. Prot (Ed.), Référentiel, Compétences, Développement. (pp. 119-132). Toulouse: Octarès.

Brough, J.E., Schwartz, M., Gupta, S.K., Anand, D.K., Kavetsky, R., \& Pettersen, R. (2007). Towards the development of a virtual environment-based training system for mechanical assembly operations. Virtual Reality, 11, 189-206.

Cau-Bareille, D. (2011). Factors influencing early retirement in a femal-dominated profession: Kindergarten teacher in France. Work, 40(Suppl.), 15-30.

Cau-Bareille, D., Gaudart, C., \& Delgoulet, C. (2012). Training, age and technological change : difficulties associated with age, the design of tools, and the organization of work. Work, 41(2), 127-141.

Chatigny, C., Levesque, S. \& Riel, J. (2012). Training yourself while training students: The constant challenge of vocational training teachers. Work, 41(2), 143-153.

Chatigny, C., \& Vezina, N. (1995). Analyse du travail et apprentissage d'une tâche complexe ; étude de l'affilage du couteau dans un abattoir. Le Travail Humain, 58(3), 145-162.

Chatigny, C., \& Vezina, N. (2008). L'analyse ergonomique de l'activité de travail : un outil pour développer les dispositifs de formation et d'enseignement. In Y. Lenoir (Ed.), Didactique professionnelle et didactiques disciplinaires en débat. (pp. 127-159). Toulouse: Octarès.

Clot, Y. (2000). La fonction psychologique du collectif. In H. Benchekroum \& A. Weil-Fassina (Eds.), Approches du collectif, Toulouse: Octarès.

Cloutier, E., Fournier, P.-S., Ledoux, É., Gagnon, I., Beauvais, A., \& Vincent-Genod, C. (2012). La transmission des savoirs de métier et de prudence par les travailleurs expérimentés : comment soutenir cette approche dynamique de formation dans les milieux de travail. Rapport, Montréal: Commission des partenaires du marché du travail.

Clot, Y., Faïta, D., Fernandez, G., \& Scheller, L. (2000). Entretiens en autoconfrontation croisée : une méthode en clinique de l'activité. PISTES, 2(2).

Costello, P. (1997). Health and Safety Issues associated with Virtual Reality - A Review of Current Literature, AGOCG Technical Report Series.

Daniellou, F. (1996). L'ergonomie en quête de ses principes. Toulouse: Octarès Éditions.

Daniellou, F. (2004). L'ergonomie dans la conduite de projets de conception de systèmes de travail. In P. Falzon (Ed.), Ergonomie. (pp. 359-373). Paris: PUF.

Delgoulet, C. (2001). La construction des liens entre situations de travail et situations d'apprentissage dans la formation professionnelle. PISTES, 3(2). http://www.pistes.uqam.ca

Delgoulet, C. (2012). Being a trainer in the French vocational training system: a case study on job status and working conditions related to perceived health. Work, 41(suppl.), 5203-5209.

Delgoulet, C., \& Boccara, V. (2013). Analyse du travail des monteurs - ajusteurs de l'aéronautique en production et en formation pour la conception d'un environnement virtuel de formation. Rapport intermédiaire $\left(\mathrm{n}^{\circ} 2\right)$ Nikita, LATI - ANR, janvier.

Delgoulet, C., Largier, A., \& Tirilly, G. (2013). La mesure des tutorats en entreprise : enjeux, complexité et limites. Formation - Emploi, 124, 45-62.

Delgoulet, C., \& Marquié, J.-C. (2002). Age differences in learning maintenance skills: a field study. Experimental Aging Research, 28, 25-37.

Delvolvé, N., \& Davila W. (1994). Effets de la semaine de classe de quatre jours sur l'élève : Les rythmes scolaires en Europe, Enfance, 4, 400-407.

Desnoyers, L., Berthelette, D., Gourde, D., \& Authier, M. (1997). Évaluation d'un programme de formation en santé et en sécurité du travail. Actes du 32ème congrès de la SELF : Recherche, 
pratique et formation en ergonomie (pp. 335-346). Lyon: Editions GERRA.

Faïta, D. (2003). Apport des sciences du travail à l'analyse des activités enseignantes. Skhôlé, hors série 1, 17-23.

Falzon, P., \& Mollo, V. (2004). Auto- and allo-confrontation as tools for reflective activities. Applied Ergonomics, 35, 531-540.

Falzon, P., \& Teiger, C. (2011). Ergonomie et formation. In, P. Carré \& P. Caspar (Eds.), Le traité des sciences et techniques de la formation (pp. 145-162), Paris: Dunod.

Gaudart, C. (2000). Conditions for maintaining ageing operators at work - a case study conducted at an automobile manufacturing plant. Applied Ergonomics, 31(5), 453-462

Gaudart, C., Delgoulet, C., \& Chassaing, K. (2008). La fidélisation de nouveaux dans une entreprise du BTP : Approche ergonomique des enjeux et des déterminants. Activités, 5(2), 2-24. http://www.activites.org/ v5n2/v5n2.pdf

Gaudart, C., Petit, J., Dugué, B., Daniellou, F., Davezies, P., \& Théry, L. (2012). Impacting working conditions through trade union training. WORK, 41(2), 165-175.

Guérin, F., Laville, A., Daniellou, F., Duraffourg, J., \& Kerguelen, A. (1991). Comprendre le travail pour le transformer. Lyon: Éditions ANACT.

Guilbert, L., \& Lancry, A. (2007). L'analyse des activités des cadres : l'intérêt de la triangulation des méthodes. Le Travail Humain, 70(4), 313-342.

Haradji, Y., Kostulski, K., Morais, A., \& Ughetto, P. (2014). Éditorial. Activités, 11(2), 1-2. http://www.activites.org/v11n2/V11n2.pdf

Hu, B., Zhang, W., \& Salvendy, G. (2012). Impact of Multimodal Feedback on Simulated Ergonomic Measurements in a Virtual Environment: A Case Study with Manufacturing Workers. Human Factors and Ergonomics in Manufacturing and Service Industries, 22(2), 145-155.

Hubault, F., Noulin, M., Dupont, F., \& Mélier, B. (1994). Formation par et pour l'action : exemples d'apprentissage de la conduite de projet intégrant le point de vue du travail. Actes du congrès IEA'94 (pp. 71-73). Toronto (Canada), 15-19 Août 1994.

Jacobs, K., \& Backer, N.A. (2002). The association between children's computer use and musculoskeletal discomfort. Work, 18(2), 221-226

Jeantet, A. (1998). Les objets intermédiaires dans les processus de conception de produits. Sociologie du travail, 98(3), 291-316.

Jick, T. D. (1979). Mixing qualitative and quantitative methods: triangulation in action. Administrative Science Quarterly, 24(4), 602-611.

Jobert, G. (1987). Une nouvelle professionnalité pour les formateurs d'adultes. Éducation Permanente, 87, 19-33

Jobert, G. (1993). Les formateurs et le travail : chronique d'une relation malheureuse. Éducation Permanente, 116, 7-18

Jobert, G. (2000). Dire, penser, faire. À propos de trois métaphores agissantes en formation des adultes. Éducation Permanente, 143, 7-28.

Lacomblez, M., Bellemare, M., Chatigny, C. Delgoulet, C., Re, A., Trudel, L., \& Vasconcelos, R. (2007). Ergonomics analysis of work activity and training: basic paradigm, evolutions and challenges. In R. Pikaar, E. Konongsveld, \& P. Settels (Eds.) Meeting Diversity in Ergonomics (129-142). Elsevier Ltd.

Lang, N. (2013). Évaluation d'une formation à l'analyse ergonomique du travail pour des enseignants de lycée professionnel. In C. Teiger \& M. Lacomblez (Eds.), (Se) former pour transformer le travail. Dynamique de constructions d'une analyse critique du travail (pp. 582-593), Laval Québec: PUL.

Leplat, J. (1955). Analyse du travail et formation. Bulletin du CERP, 4(9), 175-184

Leplat, J. (2002). De l'étude de cas à l'analyse de l'activité. PISTES, 4(2).

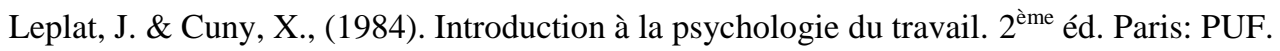

Leppänen, A. (2001). Improving the mastery of work and the development of the work process in paper production. Industrial Relations, 56(3), 576-609.

Mayen, P. (1999). Des situations potentielles de développement. Éducation Permanente, 139, 65-86. 
Mayen, P., Métral, J.-F., \& Tourmen, C. (2010). Les référentiels en formation : enjeux, légitimité, contenu et usage. Recherche et formation, 64, 31-46.

Méhaut, P. (1995). Le rôle de l'entreprise dans la production des qualifications : effets formateurs de l'organisation du travail. Luxembourg: Office des Publications des Communautés Européennes.

Messing, K., Escalona E., \& Seifert, A.M. (1996). La minute de 120 secondes, analyse du travail des enseignantes de l'école primaire. Rapport Centre D'enseignement du Québec - CINBIOSE.

Montmollin (de), M. (1974). L'analyse du travail : Préalable à la formation. Paris: Armand Colin.

Montmollin (de), M. (1980). Ergonomie et organisation du travail. In J. Leplat (Ed.), L'analyse du travail (Vol. 1). Toulouse: Octarès.

Montreuil, S., Brisson, C., Arial, M., \& Trudel, L. (1997). Évaluation des effets d'un programme de formation chez les utilisateurs de terminaux à écran de visualisation. Rapport de recherche de l'IRSST R-167, Montréal: IRSST.

Nicolas, L. (2000a). L'activité de simulation en analyse fonctionnelle : vers des outils anthropocentrés pour la conception de produits automobiles. Thèse de Doctorat en Ergonomie. Paris: CNAM.

Nicolas, L. (2000b). La simulation Langagière en analyse fonctionnelle : entre travail des concepteurs et travail des ergonomes. Actes du 35ème congrès de la SELF : Communication et Travail (pp. 178-187). Toulouse. http://www.ergonomie-self.org/documents/35eme-Toulouse2000/conception.pdf

Olry, P., \& Vidal-Gomel, C. (2011). Conception de formation professionnelle continue : tensions croisées et apports de l'ergonomie, de la didactique professionnelle et des pratiques d'ingénierie. Activités, 8(2), 115-149. http://www.activites.org/v8n2/v8n2.pd

Ombredane, A., \& Faverge, J.M. (1955). L'analyse du travail. Facteur d'économie humaine et de productivité. Paris: PUF.

Ouellet, S., \& Vézina, N. (2008) Savoirs professionnels et prévention des TMS : réflexions conceptuelles et méthodologiques menant à leur identification et à la genèse de leur construction. PISTES, 10(2), www.pistes.uqam.ca/v10n2/articles/v10n2a5.html

Ouellet, S., \& Vézina, N. (2009). Savoirs professionnels et prévention des TMS : portrait de leur transmission durant la formation et perspectives d'intervention. PISTES, 11(2). http://www.pistes.uqam.ca/v11n2/articles/v11n2a4.htm

Ouellet, S., Vézina, N., Chartrand, J., Perrier P.-P., \& Malo J.-L. (2003). L’implantation de la rotation de postes : un exemple de démarche préalable. PISTES, 5(2). www.pistes.uqam.ca/v5n2/articles/v5n2a14.htm

Ouellet, S., Vézina, N., \& Chatigny, C. (2013). Transformer les conditions d'apprentissage par la transformation des conditions de travail du formateur. Colloque international. Les questions vives en éducation et formation : regards croisés France-Canada. Nantes: CREN.

Pacaud, S. (1975). Le travailleur vieillissant : quelques réflexions sur ses difficultés, mais aussi ses facilités d'adaptation au travail. In A. Laville, C. Teiger, \& A. Wisner, (Eds.), Age et Contraintes de Travail. Aspects sociologiques, psychologiques, physiologiques (pp. 115-179), Paris: NEB Editions Scientifiques.

Pastré, P. (1999). La conceptualisation dans l'action : bilan et nouvelles perspectives. Éducation permanente, 139, 13-35.

Pastré, P. (2005). Dynamique et métamorphose des compétences professionnelles. Psychologie du travail et des organisations, 11, 73-87.

Pastré, P. (2011). La didactique professionnelle : approche anthropologique du développement chez les adultes. Paris: PUF.

Rabardel, P. (1995). Les hommes et les technologies. Paris: Armand Collin.

Rabardel, P., Teiger, C., Laville, A., Rey, P., \& Desnoyers, L. (1991). Ergonomic work analysis and training. In Y. Quéinnec, \& F. Daniellou (Eds.), XIth Congress of International Ergonomics Association, Designing for everyone (pp. 1738-1740). London: Taylor and Francis.

Rabardel, P., Carlin, N., Chesnais, M., Lang, N., \& Pascal, M. (1998). Ergonomie concepts et méthodes. Toulouse: Octarès Editions.

Rabardel, P., \& Six, B. (1995). Outiller les acteurs de la formation pour le développement des 
compétences au travail. Éducation permanente, 123(2), 33-46.

Ria, L., Sève, C., Durand, M., \& Bertone, S. (2004). Indétermination, contradiction, exploration : trois expériences typiques des enseignants débutants en éducation physique. Revue des sciences de l'éducation, 30(3), 535-554.

Riel, J., \& Messing, K. (2011). Counting the minutes: Administrative control of work schedule and time management of secondary school teachers in Québec, Work, 40(1), 59-70.

Rogalski, J. (2003). Y a-t-il un pilote dans la classe ? Une analyse de l'activité l'enseignant comme gestion d'un environnement dynamique ouvert. Recherche en didactique des mathématiques, 23, 343-388.

Rogalski, J. (2004). La didactique professionnelle : une alternative aux approches de « cognition située » et « cognitiviste » en psychologie des acquisitions. Activités, 1(2), 103-120. http://www.activites.org/v1n2/Rogalski.pdf.

Rogalski, J. (2012). Théorie de l'activité et didactique, pour l'analyse conjointe des activités de l'enseignant et de l'élève. Jornal Internacional de Estudos em Educaçao Metamatica, 5(1), 1-37.

Samurçay, R., \& Rabardel, P. (2004). Modèles pour l'analyse de l'activité et des compétences, propositions. In R. Samurçay \& P. Pastré (Eds/), Recherches en didactique professionnelle (pp. 163-180). Toulouse: Octarès.

Samurçay, R., \& Rogalski, J. (1998). Exploitation didactique des situations de simulation. Le Travail Humain, 61(4), 333-359.

Santos, M., \& Ferreira, A. (2012). Conditions, demands and constraints on trainers' activity: a case study in the Portuguese context. Work, 41(suppl.), 5210-5217

Santos, M., \& Lacomblez, M. (2007). Que fait la peur d'apprendre dans la zone prochaine de développement ? Activités, 4(2), 16-29. http://www.activites.org/v4n2/santos.pdf

Teiger, C., \& Lacomblez, M. (Eds) (2013). (Se) former pour transformer le travail. Dynamique de constructions d'une analyse critique du travail. Laval Québec: PUL.

Teiger, C., \& Laville, A. (1991). L'apprentissage de l'analyse ergonomique du travail, outil d'une formation pour l'action. Travail et emploi, 47, 53-62.

Teiger, C., \& Montreuil, S. (1996). The foundations and contributions of ergonomics work analysis in training programs, Safety Science, 23(2/3), 81-95.

Thébault, J. (2013). La transmission professionnelle : processus d'élaboration d'interactions formatives en situation de travail. Une recherche auprès de personnels soignants dans un Centre Hospitalier Universitaire. Thèse de doctorat, CNAM, Paris.

Thébault, J., Delgoulet, C., Fournier, P. S., Gaudart, C., \& Jolivet, A. (2014). La transmission à l'épreuve des réalités du travail. Éducation permanente, 198, 85-99.

Thébault, J., Gaudart, C., Cloutier, E., \& Volkoff, S. (2012). Transmission of vocational skills between experienced and new hospital workers. Work, 41(1), 195-204.

Veyrac, H., \& Asloum, N. (2009). Les tâches appropriées des professeurs d' enseignement professionnel. Illustration du hiatus entre travail en entreprise et formation. Activités, 6(1), 69-86. http://www.activites.org/v6n1/v6n1.pdf

Viau-Guay, A. (2014). L'intégration des savoirs théoriques, pratiques et d'autorégulation chez les apprenants : analyse de l'activité d'enseignants en formation professionnelle et contribution à la formation à l'enseignement. Activités, 11(2), 88-111, http://www.activites.org/v11n2/v11n2.pdf

Vidal-Gomel, C. (2001). Le développement des compétences pour la gestion des risques professionnels. Le domaine de la maintenance des systèmes électriques. Thèse de Doctorat, Université: Paris 8, St-Denis.

Vidal-Gomel, C., Boccara, V., Rogalski, J., \& Delhomme, P. (2012). Sharing the driving-course of a same trainee between different trainers, what are the consequences? Work: A Journal of Prevention, Assessment, \& Rehabilitation, 41, 205-215.

Vinck, D. (2009). De l'objet intermédiaire à l'objet-frontière. Revue d'anthropologie des connaissances, 3(1), 51-72.

Weill-Fassina, A., \& Pastré, P. (2004). Les compétences professionnelles et leur développement. In P. Falzon (Ed.), Ergonomie (pp. 213-232). Paris: PUF.

Wendelen, E. (1996). Training trade unionists in ergonomics: interaction between public policies, 
trade union practices and development of research. Safety Science, 23(2:3), 107-117.

\section{RESUME}

Cet article présente et discute une démarche d'analyse "des travails» dans le cadre d'un projet de conception d'un environnement virtuel de formation (EVF) au métier d'assembleurmonteur en aéronautique. Cette démarche, à la croisée de l'ergonomie et de la didactique professionnelle, a été menée en trois phases : 1) une analyse de la demande et du projet, 2) une analyse des travails (formateurs, formés, opérateurs de production), 3) un dispositif de co-analyse des savoirs de métier et de leurs modalités de transmission, impliquant les formateurs. Au cours de cette démarche, la focale de l'analyse du travail, les acteurs impliqués ainsi que les résultats produits évoluent. À partir d'une réflexion sur notre pratique d'ergonome, il s'agit de discuter des apports d'une analyse «des travails » comme moyen d'identifier les multiples horizons des situations de formation à concevoir et d'intervention en conception.

\section{MOTS-CLES}

conception, formation, environnement virtuel, analyse des travails, aéronautique

\section{REFERENCEMENT}

Boccara, V., \& Delgoulet, C. L'analyse des travails pour la conception en formation. Contribution de l'ergonomie à l'orientation de la conception amont d'un environnement virtuel pour la formation. Activités, 12(2), 73-97. http://www.activites.org/v12n2/V12n2.pdf

Article soumis le 29 juillet 2014, accepté le 18 juin 2015 